\title{
SHOCK FORMATION IN ELECTRON-ION PLASMAS: MECHANISM AND TIMING
}

\author{
A. Stockem Novo ${ }^{1}$, A. Bret ${ }^{2,3}$, R. A. Fonseca ${ }^{4,5}$, and L. O. Silva ${ }^{4}$ \\ ${ }^{1}$ Institut für Theoretische Physik, Lehrstuhl IV: Weltraum- and Astrophysik, Ruhr-Universität, Bochum, Germany; anne@tp4.rub.de \\ ${ }^{2}$ ETSI Industriales, Universidad de Castilla-La Mancha, E-13071 Ciudad Real, Spain \\ ${ }^{3}$ Instituto de Investigaciones Energéticas y Aplicaciones Industriales, Campus Universitario de Ciudad Real, E-13071 Ciudad Real, Spain \\ ${ }^{4}$ GoLP/Instituto de Plasmas e Fusão Nuclear, Instituto Superior Técnico, Universidade de Lisboa, Lisboa, Portugal \\ ${ }^{5}$ DCTI, ISCTE-Lisbon University Institute, Portugal \\ Received 2015 March 12; accepted 2015 April 1; published 2015 April 22
}

\begin{abstract}
We analyze the full shock formation process in electron-ion plasmas in theory and simulations. It is accepted that electromagnetic shocks in initially unmagnetized relativistic plasmas are triggered by filamentation instability. However, the transition from the first unstable phase to the quasi-steady shock is still missing. We derive a theoretical model for the shock formation time, taking into account filament merging in the nonlinear phase of the filamentation instability. This process is much slower than in electron-positron pair shocks, and so the shock formation is longer by a factor proportional to $\sqrt{m_{i} / m_{e}} \ln \left(m_{i} / m_{e}\right)$.
\end{abstract}

Key words: instabilities - relativistic processes - shock waves

\section{INTRODUCTION}

Collisionless shocks are ubiquitous in astrophysical environments such as gamma-ray bursts, active galactic nuclei, or pulsar wind nebulae. They are especially important in the context of cosmic ray acceleration. Once the shock is in a quasi-steady state, the jump conditions can be determined from the conservation of mass, energy, and momentum in a fluid model (Blandford \& McKee 1976). The density jump from upstream to downstream of a relativistic strong shock is given in two dimensions by $n_{2} / n_{1} \approx 3$. Plasma instabilities are the mediators of such collisionless shocks (Sagdeev 1966), but it is still not known precisely how the transition from the initial plasma turbulence to the final quasi-steady state of the shock happens.

In a symmetric counterstreaming flow of charged particles, plasma instabilities develop causing an isotropization of the particle momenta and initiating the deceleration of the plasma flows. In relativistic, cold, and initially unmagnetized plasmas, electromagnetic current filamentation modes which inhabit a strong perpendicular component are dominant (Fried 1959; Weibel 1959). The energy is transferred from an initial longitudinal streaming into perpendicular directions. The particle forward motion is slowed down and a collisionless shock begins to form with a density ratio of $n_{2} / n_{1}>2$.

Since nonlinear processes are involved, particle-in-cell (PIC) simulations are an excellent tool to investigate the shock formation process (Hededal et al. 2005; Medvedev et al. 2005; Spitkovsky 2008; Martins et al. 2009; Nishikawa et al. 2011; Fiúza et al. 2012; Sironi et al. 2013; Stockem et al. 2014; Huntington et al. 2015). In a previous study, we identified the shock formation time of electron-positron pair shocks as twice the saturation time of the magnetic field amplification due to filamentation instability, $\tau_{f, e}=2 \tau_{s, e}$ (Bret et al. 2013, 2014). Surprisingly, electron-ion shocks do not show the same feature as one would expect from the rapid relativistic mass increase of the electrons. Our analysis shows a shock formation time of $\tau_{f, i} \approx 3 \sqrt{m_{i} / m_{e}} \tau_{f, e}$. However, we observed a similarity between both scenarios: the steady-state shock is formed when the first ions start to recirculate. The nonlinear phase is faster in pair shocks and no additional time is necessary for the merging of magnetic flux tubes, and so the recirculation process starts earlier. Consequently, particles are mainly scattered in the turbulence rather than performing a full gyro rotation, which is why the isotropization process is less effective. We present a detailed theory for shock formation in electron-ion plasmas and compare it to state-of-the-art PIC simulations.

\section{SHOCK FORMATION TIME}

We consider a simple scenario for shock formation with the plasma initially being unmagnetized and symmetric counterstreaming relativistic beams of electrons and ions of mass ratio $m_{i} \geqslant m_{e}$ and Lorentz factor $\gamma_{0}$. We refer to pair shocks of electrons and positrons when $m_{i}=m_{e}$. The beams are initially cold, characterized by the temperature parameter $\mu=\gamma_{0} m c^{2} / k_{B} T$, where the mass $m$ and temperature $T$ refer to the respective species. Such scenarios are a stimulating environment for the current filamentation instability (Fried 1959) to occur. The growth rate of the cold current filamentation instability, for electron beams as well as for ion beams, is given by $\delta=\sqrt{2 / \gamma_{0}} \beta_{0} \omega_{p}$, where $\beta_{0}=v_{0} / c$ denotes the normalized fluid velocity of the beam and the plasma frequency is given by $\omega_{p}=\sqrt{4 \pi n_{0} e^{2} / m}$ with the initial uniform beam density $n_{0}$ (Bret et al. 2010).

Let us start by describing the mechanisms at work that lead to shock formation before we turn to the analytical evaluation of the shock formation time. As has already been highlighted by various authors (Lyubarsky \& Eichler 2006; Shaisultanov et al. 2012; Davis et al. 2013), the electron Weibel instability is first triggered when the two plasmas start overlapping. By the time it saturates, the instability has generated filaments of the size of the electron Larmor radius in the field at saturation (Bret et al. 2013). By the time the ion Weibel instability starts to grow, an unstable wavelength has already been seeded. This wavelength corresponds precisely to that which resulted from the merging of the filaments associated with the electron Weibel instability with a typical length scale of $c / \omega_{p e}$. Consequently, by the time the ion Weibel instability saturates, the field is near equipartition with the ions but the filaments are still the size of the electronic Larmor radius, not the ion radius, since the instability of the ions was initially seeded at these 
length scales. Such filaments are too small to efficiently deflect the ion flow and need to merge in order to reach the required size (Milosavljevic et al. 2006; Chang et al. 2008; Spitkovsky 2008; Davis et al. 2013). Once the appropriate number of merging events has been achieved, the filaments reach the size of the ion Larmor radius. Only then is the ion flow deflected enough for the shock to start forming. Assuming that this happens at a time $\tau$ and following the reasoning explained in Bret et al. (2014), the shock formation time will be $2 \tau$ in twodimensional (2D) space, and $3 \tau$ in three-dimensional (3D) space.

\subsection{Saturation Phase}

The electron Weibel instability grows first, amplifying the field from its fluctuation value up to nearly equipartition with the electrons (Bret et al. 2013). The saturation time here is given by

$$
\tau_{s, e}=\delta_{e}^{-1} \Pi \omega_{p e}^{-1},
$$

where $\Pi$ is the number of $e$-foldings of the instability and $\delta_{e} \omega_{p e}^{-1}$ is its growth rate. By this time, the field has grown to nearly equipartition with (Medvedev \& Loeb 1999; Silva et al. 2003)

$$
B_{s, e}^{2}=8 \pi \gamma n_{0} m_{e} c^{2}
$$

From the cyclotron frequency $\omega_{B_{s, e}}=q B_{s, e} / \gamma m_{e} c$, we derive the size of the filaments at saturation, which is also the electronic Larmor radius in $\boldsymbol{B}_{s, e}$ (we set $\left.v_{0} \sim c\right)$ :

$$
L_{s, e}=\frac{c}{\omega_{B_{s, e}}}=\sqrt{\frac{\gamma}{2}} \frac{c}{\omega_{p e}} .
$$

We still lack a full quantitative understanding of the saturation magnetic field and this will be explored elsewhere ( $\mathrm{K}$. Schoeffler et al. 2015, in preparation).

Contrary to the electron current filamentation instability, which has to amplify the plasma thermal fluctuations, the ion Weibel instability finds an unstable mode already seeded and further amplifies it, growing preferentially at that wavenumber. This is clear from the fact that the growth rate of the ion Weibel instability depends slowly on $k$ for wavenumbers $k \geqslant \frac{\omega_{p e}}{c}\left[1+\left(\frac{m_{e}}{4 m_{i}}\right)^{1 / 3}\right]^{-3 / 2}$ (Davidson 1972). At saturation, the ion Weibel instability has grown the field to

$$
B_{s, i}^{2}=8 \pi \gamma n_{0} m_{i} c^{2},
$$

while the size of the filaments is still given by Equation (3). The growth time of the ion phase of the instability is

$$
\tau_{s, i}=\delta_{i}^{-1} \ln \left(\frac{B_{s, i}}{B_{s, e}}\right) \omega_{p i}^{-1}=\delta_{i}^{-1} \ln \sqrt{\frac{m_{i}}{m_{e}}} \omega_{p i}^{-1} .
$$

The total duration of this saturation phase is $\tau_{s}=\tau_{s, e}+\tau_{s, i}$, and using $\delta \equiv \delta_{e}=\delta_{i} \sqrt{m_{i} / m_{e}}$ we obtain

$$
\tau_{s}=\delta^{-1} \ln \sqrt{\frac{m_{i}}{m_{e}}}\left(\frac{\Pi \sqrt{\frac{m_{e}}{m_{i}}}}{\ln \sqrt{\frac{m_{i}}{m_{e}}}}+1\right) \omega_{p i}^{-1} .
$$

Considering a number of $e$-foldings $\Pi \sim 20$, we find that even for an artificially low mass ratio of 100, the term $\Pi \sqrt{m_{e} / m_{i}} / \ln \sqrt{m_{i} / m_{e}} \ll 1$. Consequently, the duration of the entire saturation phase simply reads $\tau_{s} \sim \tau_{s, i}$. In reality, the ion growth rate $\delta_{i}$ is lower than $\delta_{e} \sqrt{m_{e} / m_{i}}$, that is, the growth rate of the cold ion current filamentation instability, because it grows over a background of hot electrons. However, the consequences are negligible (see Section 2.3).

\subsection{Filament Merging Phase}

The dynamics of filament merging were studied previously in Medvedev et al. (2005), who considered a simple 2D model of infinitely long cylindrical filaments of radius $D / 2$, spaced at a distance $D .^{6}$

The filaments need to merge $n$ times in order to grow from the electronic Larmor radius (3) to the ionic radius. According to Medvedev et al. (2005), the expression for the merging time is different whether the transverse motion is relativistic or not (merging of the filaments implies a motion transverse to the flow). At any rate, the maximum transverse velocity achieved during one merging reads (Medvedev et al. 2005)

$$
v_{m}=\frac{1.67}{4} \frac{D \omega_{p i}}{\sqrt{\gamma}}
$$

considering $v_{0} \sim c$. We now compare this velocity to $c$. For the merging motion to be non-relativistic, we would need

$$
v_{m} \ll c \Rightarrow D \ll 2.4 \sqrt{\gamma} \frac{c}{\omega_{p i}} .
$$

If this condition is fulfilled at the end of the merging phase, then it is always fulfilled because filaments grow with time. Therefore, if we replace $D$ by the ion Larmor radius $L_{i}=\sqrt{\gamma / 2} c / \omega_{p i}$, then we find that the above condition is always satisfied.

For the regime of non-relativistic transverse merging, Medvedev et al. (2005) established that all merging events take approximately the same time:

$$
\tau_{0}=2^{3 / 2} \sqrt{\gamma} \omega_{p i}^{-1} .
$$

This number now has to be multiplied by the total number $n$ of merging events. Since the initial and final filament sizes are the electron and ion Larmor radii, respectively, $n$ is given by (Medvedev et al. 2005)

$$
L_{i}=2^{n / 2} L_{e} \Rightarrow n=2 \frac{\ln \left(L_{i} / L_{e}\right)}{\ln 2}=\frac{\ln \left(m_{i} / m_{e}\right)}{\ln 2} .
$$

The total duration of the merging phase is therefore given by

$$
\tau_{m}=n \tau_{0}=\frac{2^{3 / 2}}{\ln 2} \ln \left(m_{i} / m_{e}\right) \gamma^{1 / 2} \omega_{p i}^{-1}
$$

\subsection{Shock Formation Time}

We can now proceed to the calculation of the full shock formation time. As soon as the filaments are large enough, the

\footnotetext{
6 The notation in this paper differs from that used in Medvedev et al. (2005) The correspondence is obtained by replacing the quantity $I_{0} / \sqrt{\mu_{0}}$, found in Medvedev et al. (2005), with $D \omega_{p i} v_{0} / 4 \sqrt{\gamma}$.
} 
ions are efficiently deflected by the field over a distance close to the ion Larmor radius. This happens at a time $\tau_{s}+\tau_{m}$. Then, assuming $\delta^{-1}=\sqrt{\gamma / 2}$ (Bret et al. 2010), we find

$$
\begin{aligned}
\tau_{s}+\tau_{m} & =\left(\frac{1}{2^{3 / 2}}+\frac{2^{3 / 2}}{\ln 2}\right) \gamma^{1 / 2} \ln \frac{m_{i}}{m_{e}} \omega_{p i}^{-1} \\
& \simeq 4.43 \gamma^{1 / 2} \ln \frac{m_{i}}{m_{e}} \omega_{p i}^{-1} .
\end{aligned}
$$

We thus find that the merging time is longer than the ion Weibel saturation time by a factor of $2^{3} / \ln 2 \sim 11.5$. Consequently, the uncertainty on the ion Weibel growth rate does not significantly affect the formation time. In order to find out whether or not the flow is stopped in the overlapping region by $\tau_{s}+\tau_{m}$, we need to compare $L=2 c\left(\tau_{s}+\tau_{m}\right)$, that is, the size of the region at this time, with the ion Larmor radius $L_{i}$. It is straightforward to show that

$$
L / L_{i}=8.83 \sqrt{2} \ln \frac{m_{i}}{m_{e}} \gg 1 .
$$

Consequently, the incoming flow is stopped in the overlapping region and the shock starts to form. The downstream density at time $\tau_{s}+\tau_{m}$ is still only twice the upstream density. In order to reach the expected density jump of three for the $2 \mathrm{D}$ case, ${ }^{7}$ the system needs to evolve another $\tau_{s}+\tau_{m}$ since the overlapping region no longer expands (Bret et al. 2014). In the 3D case, the expected density jump is $\approx 4$ so that it is necessary to wait another $2\left(\tau_{s}+\tau_{m}\right)$ to bring enough material into the central region. Note that the present 2D model of filament merging has been successfully tested against 3D PIC simulations (Medvedev et al. 2005). The shock formation time $\tau_{f, i}$ finally reads

$$
\frac{\tau_{f, i}}{d}=4.43 \gamma^{1 / 2} \ln \frac{m_{i}}{m_{e}} \omega_{p i}^{-1}
$$

where $d$ is the dimensionality of the system $(d=2$ (3) for a 2D (3D) setup). It is worth noting that the expression does not reduce to that obtained for a pair plasma by setting $m_{e}=m_{i}$ (Bret et al. 2013, 2014). The reason for this is that here we neglect the electron instability phase in the end result. Finally, we note that shocks in electron-ion plasmas form much slower than in pair plasmas for two reasons: on the one hand, the instability mechanism is slower; on the other hand, the merging phase is negligible in pair plasmas because the instability already generates filaments and fields sufficiently large to deflect the flow.

\subsection{Comparison with the Pair Shock Formation Time}

To compare the shock formation times of pair and electronion shocks, Equation (1) provides the formation time $\tau_{f, e}$ for the pair case (Bret et al. 2014)

$$
\tau_{f, e}=d \tau_{s, e}=d \delta_{e}^{-1} \Pi \omega_{p e}^{-1}
$$

\footnotetext{
7 See Stockem et al. (2012) and Bret (2015) for the validity of the RankineHugoniot jump conditions in collisionless plasmas.
}

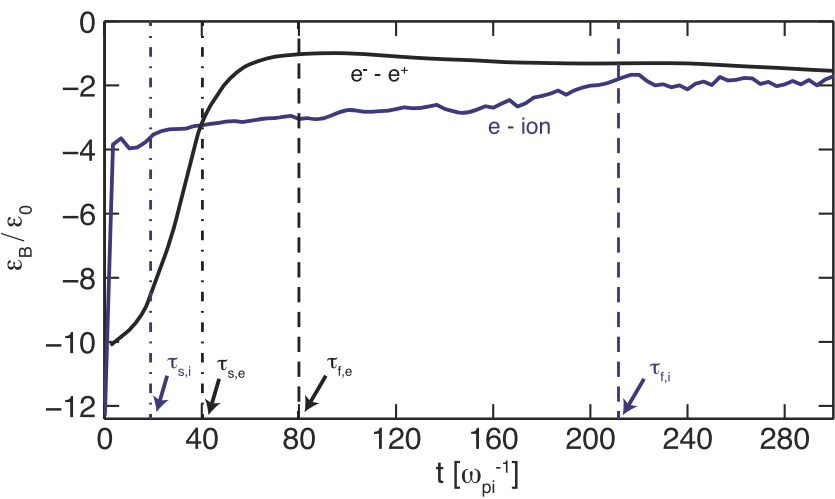

Figure 1. Normalized magnetic energy density for an electron-ion shock with $m_{i} / m_{e}=400$ (blue) and a pair shock (black) with $\gamma_{0}=25$. The lines indicate the saturation times of the filamentation instability and the shock formation times.

in terms of the dimension $d$ of the problem, whereas from Equation (14) we obtain for electron-ion shocks

$$
\frac{\tau_{f, i}}{\tau_{f, e}}=\frac{6.2}{\Pi} \ln \left(m_{i} / m_{e}\right) \sqrt{m_{i} / m_{e}} .
$$

Considering $\Pi \sim 12$, which is the value obtained in the pair shock simulations presented in the next section, we find that an electron-ion shock forms 60 times slower than a pair shock for a mass ratio of 400 , and 166 times slower for a realistic mass ratio. For a mass ratio of $400, \tau_{f, i}=3 \sqrt{m_{i} / m_{e}} \tau_{f, e}$ whereas $\tau_{f, i}=2.4 \sqrt{m_{i} / m_{e}} \tau_{f, e}$ for a mass ratio of 100 .

\section{DISCUSSION OF THE RESULTS}

Here, we present a series of 2D PIC simulations in order to validate the theoretical model. The counterstreaming beams were simulated in a simulation box with a perfectly reflecting wall in the longitudinal direction and periodic transverse boundary conditions. The bulk propagates along the $x_{1}$ axis with Lorentz factors $\gamma_{0}=25-10^{3}$, mass ratios $m_{i} / m_{e}=50-400$, and $\mu=10^{6} \gamma_{0}$. The simulation box dimensions are $L_{x}=450 \sqrt{\gamma_{0}} c / \omega_{p e}$ and $L_{y}=150 \sqrt{\gamma_{0}} c / \omega_{p e}$ with $\Delta_{x}=\Delta_{y}=0.05 \sqrt{\gamma_{0}} c / \omega_{p e}$.

The magnetic field energy density is plotted in Figure 1 to obtain information concerning the role of the filamentation instability as a mediator of the shock formation. This was done for a small slab along $x_{1}$ in a region close to the wall, which will later be a region far downstream.

In the pair shock case, the linear phase of the instabilitywhere the magnetic field grows exponentially in time and the magnetic field energy density is $\epsilon_{B} \propto \exp \left(2 \delta_{e} t\right)$ - can be clearly distinguished from the nonlinear phase where the magnetic field has saturated. The growth rate of the cold electron instability very well fits the theoretical value $\delta_{e}=0.28 \omega_{p e}$ as well as the saturation field $B_{f, e} \approx 7 m_{e} c \omega_{p e} / e$. The predicted saturation time of the filamentation instability of the pair shock $\tau_{s, e}=40 \omega_{p e}^{-1}$ and steady-state shock formation time $\tau_{f, e}=80 \omega_{p e}^{-1}$ are shown in Figure 1 and well match the simulation data.

In the case of the electron-ion shock with $m_{i} / m_{e}=400$, the evolution of the magnetic energy density shows several stages. The ion Weibel instability grows slower than 

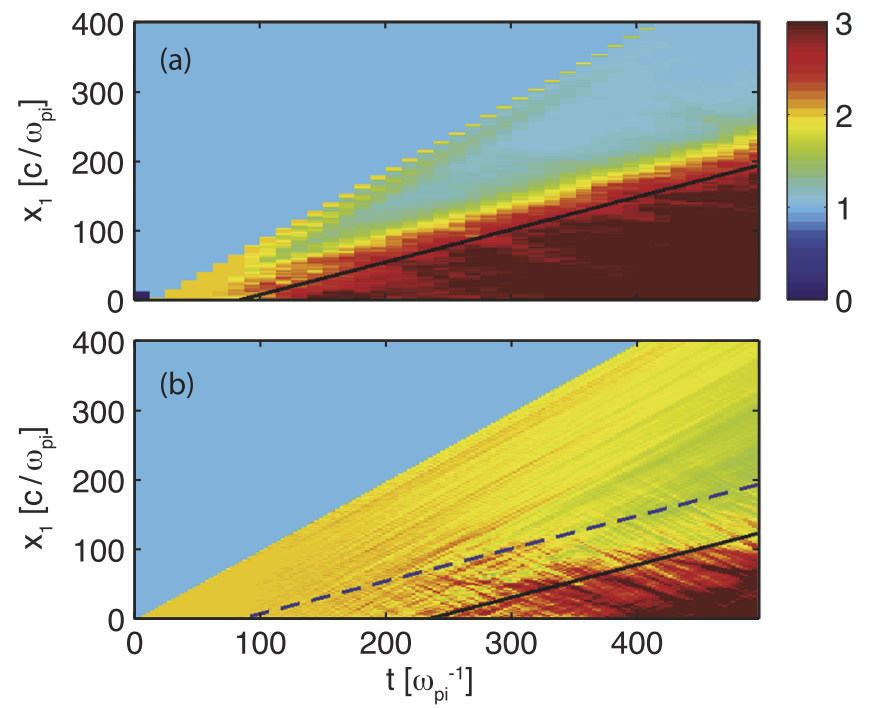

Figure 2. Ion density against space $x_{1}$ and time $t$ in units of $\omega_{p i}^{-1}=\sqrt{m_{i} / m_{e}} \omega_{p e}^{-1}$ for $\gamma_{0}=25, m_{i} / m_{e}=1$ (a) and $m_{i} / m_{e}=400$ (b). The black line indicates the shock front with $n_{2} / n_{1}=3$ and the pair shock for comparison (dashed blue).

$\delta_{i}=\sqrt{m_{e} / m_{i}} \delta_{e}=0.014 \omega_{p e}$ due to the influence of the hot electron background (Shukla et al. 2012), which is negligible when compared to the full formation time. The final magnetic field at the saturation of the ion instability approximately matches the theoretical value $B_{f, i} \approx 140 \mathrm{~cm}_{e} \omega_{p e} / e$ as well as the saturation time in the simulation $\tau_{s, i}=18 \omega_{p i}^{-1}$. The theoretical model predicts a saturation time of $13 \omega_{p i}^{-1}$ (see Equation (5)).

The black lines in Figure 2 indicate the shock front of the steady-state shock with a jump of $n_{2} / n_{1}=3$. This line is extrapolated to $x_{1}=0$ in order to define the shock formation time $\tau_{f}$ as the intersection with the time axis. For the pair shock (Figure 2(a)), $\tau_{f}$ matches the theoretical value $\tau_{f, e}=2 \tau_{s, e}$, while for the electron-ion shock we observe $\tau_{f, i}=226 \omega_{p i}^{-1} \gg 2 \tau_{s, i}=36 \omega_{p i}^{-1}$ (Figure $2(\mathrm{~b})$ ). A systematic study with different velocity and mass ratio parameters provided a factor of $2.5 \sqrt{m_{i} / m_{e}}$ with respect to the shock formation of pair shocks, which is consistent with Equation (14) (see Figure 3). The shock width imposes an uncertainty on the determination of the shock formation time, which is represented by the error bars in Figure 3.

The delayed shock formation process in electron-ion shocks due to the merging of the filaments is demonstrated in Figures 4 and 5. In both cases, the accumulation of particles becomes very effective at the time when the phase space of the different beams starts to mix (Figure 4). The first ions start to recirculate and change the sign of their longitudinal momentum. In panels (c) and (d) of Figure 4, this is demonstrated by a small fraction of particles close to zero momentum. At this stage, the magnetic field turbulent scales are large enough that the ions can finish at least half a gyro circle and the accumulation of particles becomes efficient. In pair shocks, this process occurs right after the saturation of the filamentation instability, at $t \omega_{p e}=65$, whereas in electron-ion shocks this process takes much longer. For a mass ratio of $m_{i} / m_{e}=400$, the efficient gyro reflection was observed only at $t \omega_{p i}=226$, which is slightly before the steady-state shock has formed.

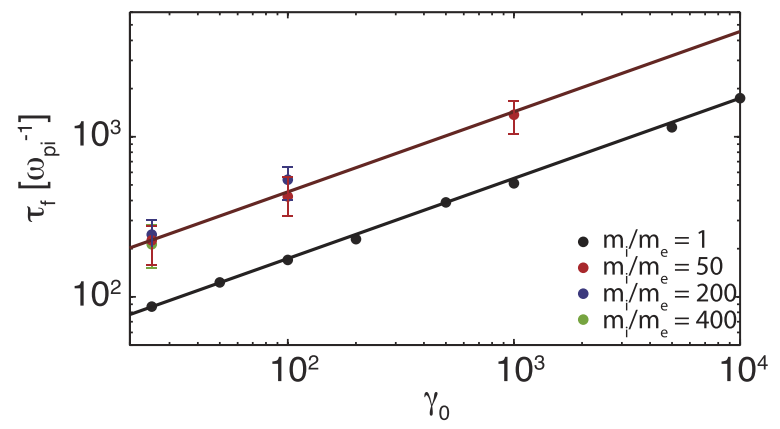

Figure 3. Shock formation time $\tau_{f, i}$ vs. $\gamma_{0}$ for a 2D pair shock with $m_{i} / m_{e}=1$ (black; Bret et al. 2014) and an electron-ion shock with different mass ratios (red). The error bars determine the uncertainty due to the finite size of the shock front.

An analysis of the magnetic field structure confirms this result. At the time when the magnetic field saturates, the transverse size of the magnetic field flux tubes is still too small to scatter the ions efficiently. The particles will feel the impact of another flux tube long before they can finish a gyro circle. For pair plasmas (Figure 5(a)), the magnetic filaments at saturation time $\tau_{s, e}=40 \omega_{p e}^{-1}$ have reached a transverse spread of $4 c / \omega_{p e}$, while the maximum magnetic field strength is of the order of $B_{3}=3 m_{e} c \omega_{p e} / e$. Particles with Lorentz factors $\gamma \leqslant 12$ have Larmor radii that fit into this scale, meaning that they can recirculate in the field before being deflected by a different flux tube. This situation is different for electron-ion plasmas. For comparison, we plotted the magnetic field structure at $\tau_{s, i}=18 \omega_{p i}^{-1}$ in Figure 5(b). At this stage, the magnetic field filaments show a transverse size on the order of $2 c / \omega_{p i}$ while $B_{3} \approx 5 m_{e} c \omega_{p e} / e$, and so only particles with nonrelativistic Lorentz factors $\gamma \sim 1$ will have Larmor radii on the same scale as the magnetic field tubes. The filaments have to undergo a further merging process as described in Equation (11) until the transverse filament size becomes the size of the ion Larmor radius for particles with $\gamma>25$.

\section{CONCLUSIONS}

We have investigated the full shock formation process in electron-ion plasmas in both theory and simulations. In contrast to electron-positron pair shocks where the shock formation time was found to be twice the saturation time of the filamentation instability, $\tau_{f, e}=2 \tau_{s, e}$, for electron-ion shocks the process is delayed to approximately $\tau_{f, i}=3 \sqrt{m_{i} / m_{e}} \tau_{f, e}$. The shock formation time is a sum of the saturation time of the instability plus an additional merging time, coming from the merging of filaments to the ion Larmor radius. At the time of the saturation of the instability, the filament size is still on the order of the electron Larmor radius. An extra time $\tau_{m}$ is thus necessary to meet the condition of shock formation, which is not the case for pair shocks. We applied the theory of Medvedev et al. (2005) to predict the merging time $\tau_{m}$. The merging time retrieved from 2D PIC simulations is in agreement with theory and the shock formation time was confirmed to be $2\left(\tau_{s, i}+\tau_{m}\right)$. Slightly before this time, the first recirculation of ions was observed. At this stage, the scale of magnetic turbulence is large enough to trigger the density compression that precedes the full shock formation.

The different scales of magnetic turbulence in electron-ion shocks compared with pair shocks could have consequences for 

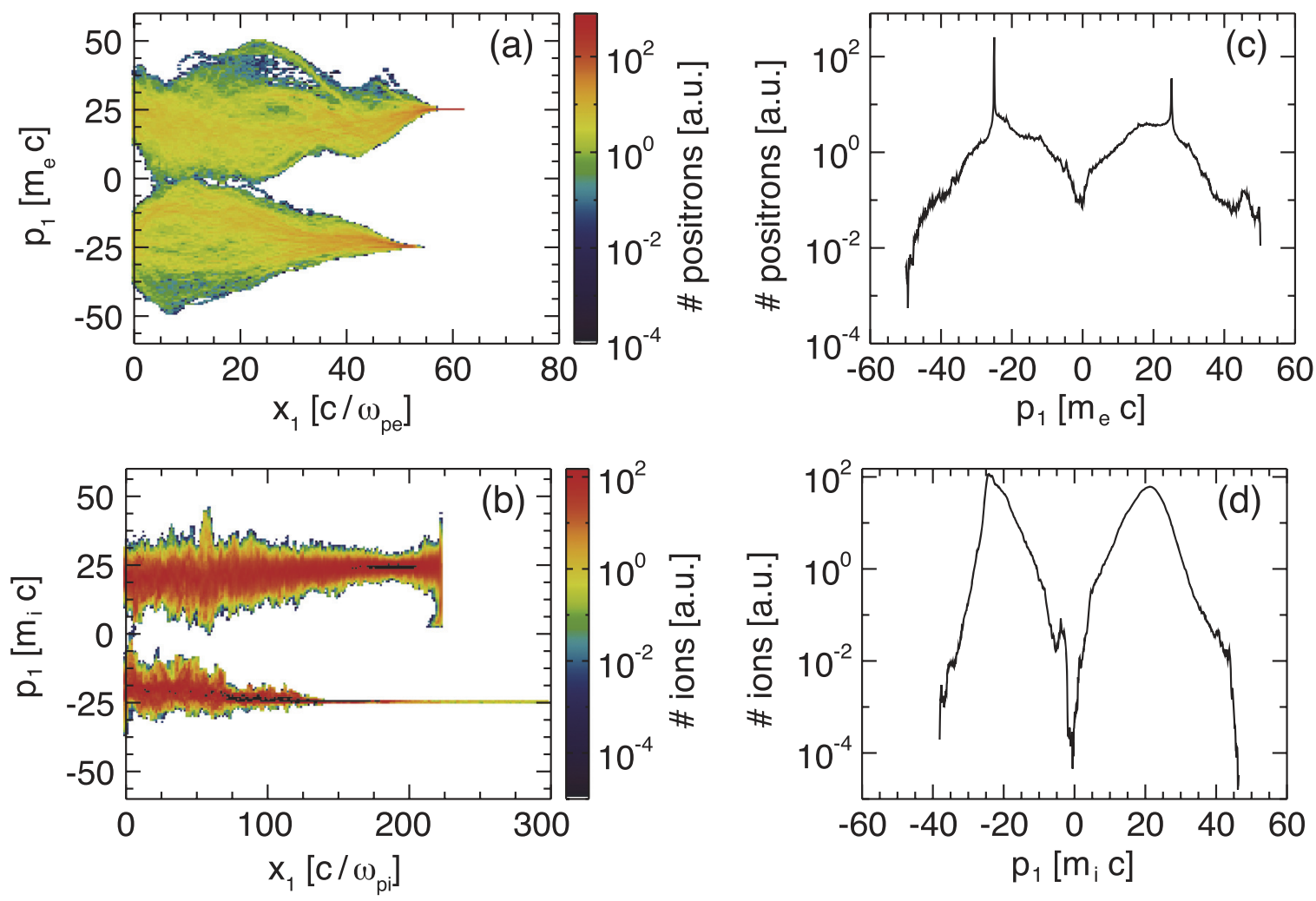

Figure 4. Momentum phase spaces of a pair shock at $65 \omega_{p e}^{-1}$ (a) and electron-ion shock with $m_{i} / m_{e}=400$ at $226 \omega_{p i}^{-1}$ (b) with respective averaged particle distributions ((c) and (d)).
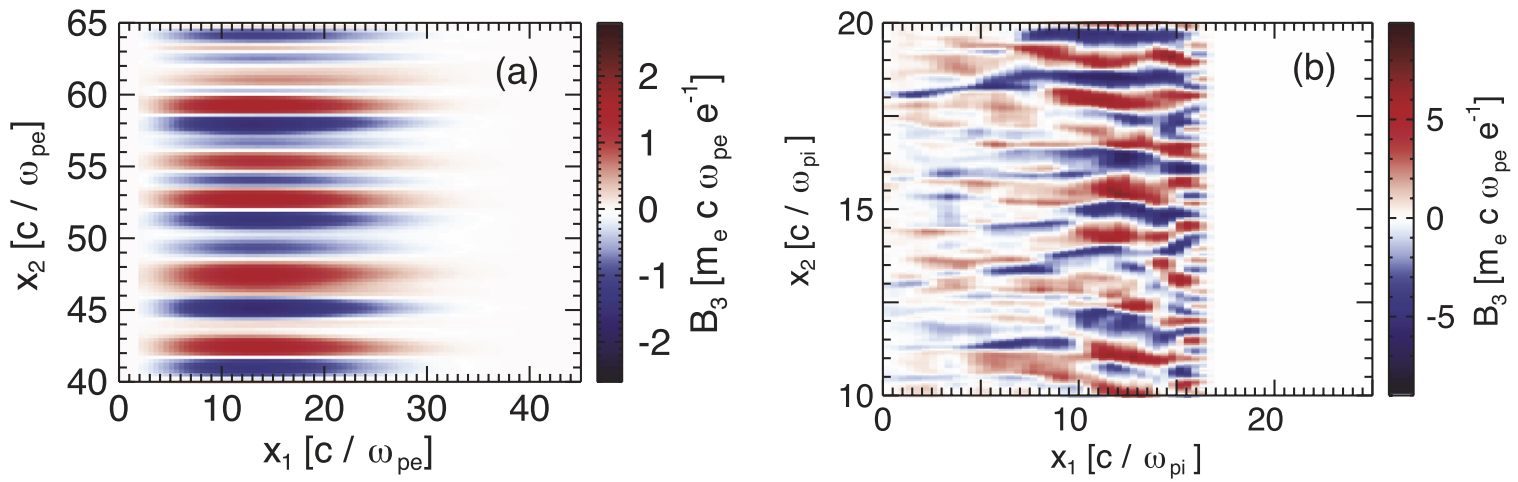

Figure 5. Magnetic field of the pair shock at saturation time $\tau_{s, e}=40 \omega_{p e}^{-1}$ (a) and for electron-ion shock at $\tau_{s, i}=18 \omega_{p i}^{-1}(\mathrm{~b})$.

the particle acceleration process. We will investigate this in our following project.

This work was supported by the European Research Council (ERC-2010-AdG grant 267841), grant ENE2013-45661-C2-1$\mathrm{P}$ from the Ministerio de Educación y Ciencia, Spain, and grant PEII-2014-008-P from the Junta de Comunidades de CastillaLa Mancha. The authors acknowledge the Gauss Centre for Supercomputing (GCS) for providing computing time through the John von Neumann Institute for Computing (NIC) on the GCS share of the supercomputer JUQUEEN at Jülich Supercomputing Centre (JSC).

\section{REFERENCES}

Blandford, R. D., \& McKee, C. F. 1976, PhFl, 19, 1130

Bret, A. 2015, arXiv: 1502.00626
Bret, A., Gremillet, L., \& Dieckmann, M. E. 2010, PhPl, 17, 120501 Bret, A., Stockem, A., Fiuza, F., et al. 2013, PhPl, 20, 042102

Bret, A., Stockem, A., Narayan, R., \& Silva, L. O. 2014, PhPl, 21, 072301

Chang, P., Spitkovsky, A., \& Arons, J. 2008, ApJ, 674, 378

Davidson, R. C. 1972, Methods in Nonlinear Plasma Theory (New York and London: Academic)

Davis, S. P., Capdessus, R., d'Humières, E., et al. 2013, HEDP, 9, 231

Fiúza, F., Fonseca, R. A., Tonge, J., Mori, W. B., \& Silva, L. O. 2012, PhRvL, 108,235004

Fried, B. D. 1959, PhFl, 2, 337

Hededal, C. B., Haugbølle, T., Frederiksen, J. T., \& Nordlund, Å 2005, Nuovo Cimento C, 28, 411

Huntington, C. M., Fiuza, F., Ross, J. S., et al. 2015, NatPh, 11, 173

Lyubarsky, Y., \& Eichler, D. 2006, ApJ, 647, 1250

Martins, S. F., Fonseca, R. A., Silva, L. O., \& Mori, W. B. 2009, ApJ, 695, L189

Medvedev, M. V., \& Loeb, A. 1999, ApJ, 526, 697

Medvedev, M. V., Fiore, M., Fonseca, R. A., Silva, L. O., \& Mori, W. B. 2005 , ApJ, 647, 1250

Milosavljevic, M., Nakar, E., \& Spitkovsky, A. 2006, ApJ, 637, 765 
Nishikawa, K.-I., Niemiec, J., Medvedev, M., et al. 2011, AdSpR, 47, 1434 Sagdeev, R. Z. 1966, RvPP, 4, 23

Shaisultanov, R., Lyubarsky, Y., \& Eichler, D. 2012, ApJ, 744, 182

Shukla, N., Stockem, A., Fiúza, F., \& Silva, L. O. 2012, JPIPh, 78, 181

Silva, L. O., Fonseca, R. A., Tonge, J. W., et al. 2003, ApJL, 596, L121

Sironi, L., Spitkovsky, A., \& Arons, J. 2013, ApJ, 771, 54
Spitkovsky, A. 2008, ApJL, 673, L39

Stockem, A., Fiuza, F., Bret, A., Fonseca, R. A., \& Silva, L. O. 2014, NatSR, 4, 3934

Stockem, A., Fiúza, F., Fonseca, R. A., \& Silva, L. O. 2012, PPCF, 54 125004

Weibel, E. S. 1959, PhRvL, 2, 83 